\title{
Fabrication of Double-Network Hydrogels with Universal Adhesion and Superior Extensibility and Cytocompatibility by One-Pot Method
}

Hui Sun, " Min Zhang, ${ }^{\ddagger, \S, ~ " M e i l i n g ~ L i u, ~}{ }^{\dagger}$ Yi Yu, ${ }^{\ddagger}$ Xinyuan $\mathrm{Xu},{ }^{\dagger} *$ and Jianshu $\mathrm{Li}^{\dagger, \ddagger} *$

${ }^{\dagger}$ College of Polymer Science and Engineering, State Key Laboratory of Polymer Materials Engineering, Sichuan University, Chengdu, 610065, China.

${ }^{\ddagger}$ State Key Laboratory of Oral Diseases, West China Hospital of Stomatology, Sichuan University, Chengdu 610041, China.

${ }^{\S}$ National Clinical Research Center for Oral Diseases, Department of Cariology and Endodontics, West China Hospital of Stomatology, Sichuan University, Chengdu 610041, China.

${ }^{\|}$H.S. and M.Z. contributed equally to this work.

*Corresponding author: X. Xu: xinyuanxu22@163.com; J. Li: jianshu_li@scu.edu.cn 

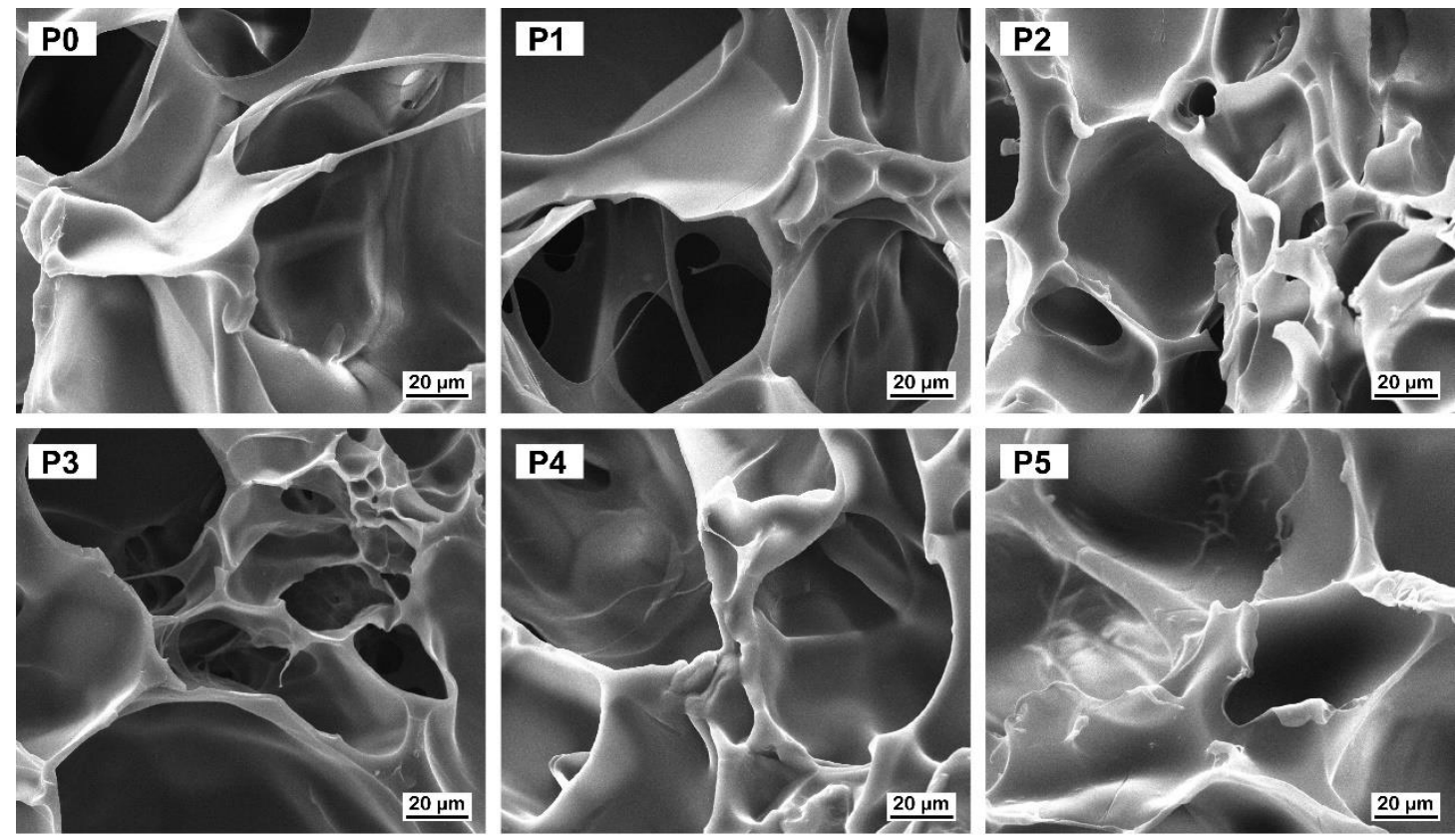

Figure S1. SEM images of diverse hydrogels without agar.
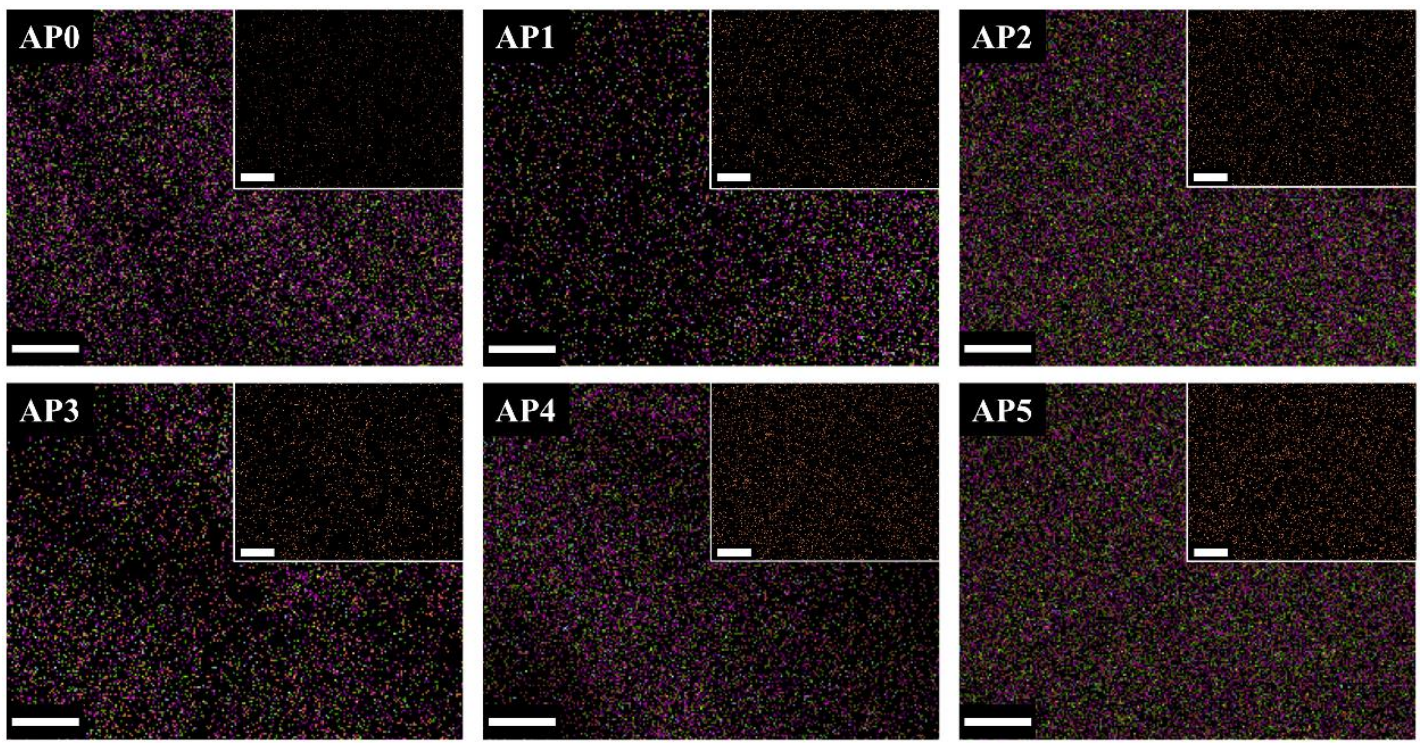

Figure S2. EDX mapping images of diverse hydrogels using field emission scanning

electron microscope (FESEM, Apreo S Hi Vac, ThermoFisher Scientific). The inset image: distribution of P element. Purple dots: C element; light blue dots: N element; green dots: O element; orange dots: P element. Scale bar $=20 \mu \mathrm{m}$. 


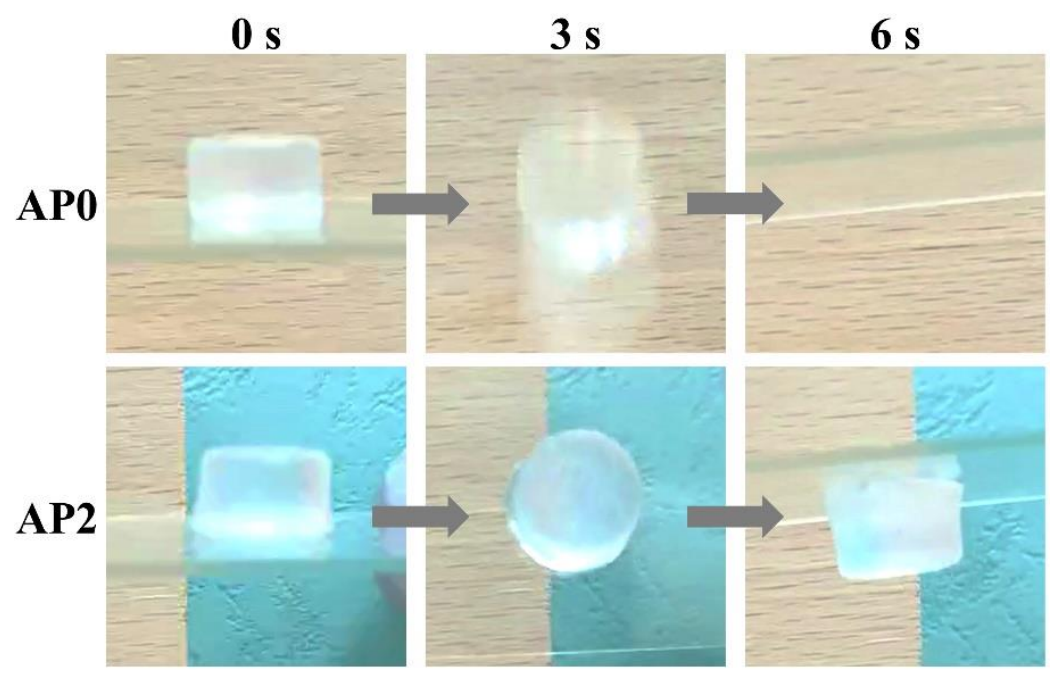

Figure S3. A series of screenshots of an overturn test video of AP0 and AP2 hydrogels.

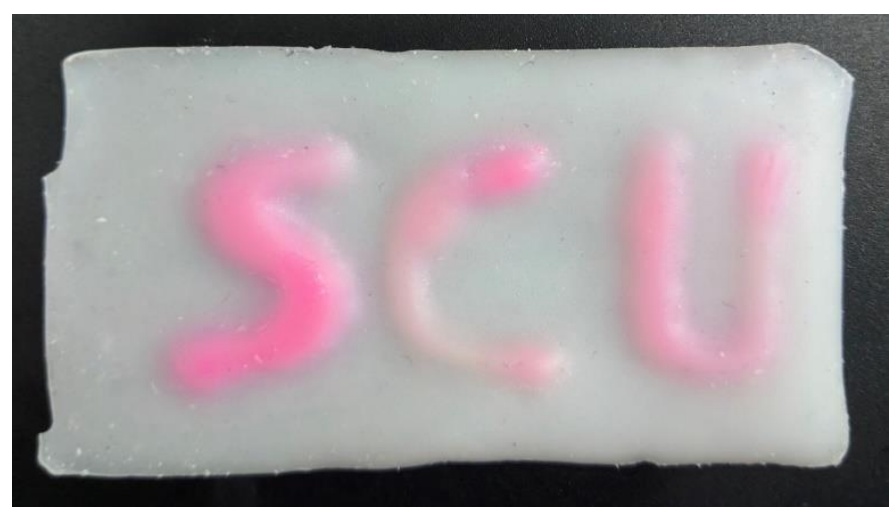

Figure S4. Optical image of injective Agar/PAM/PVPA hydrogel.

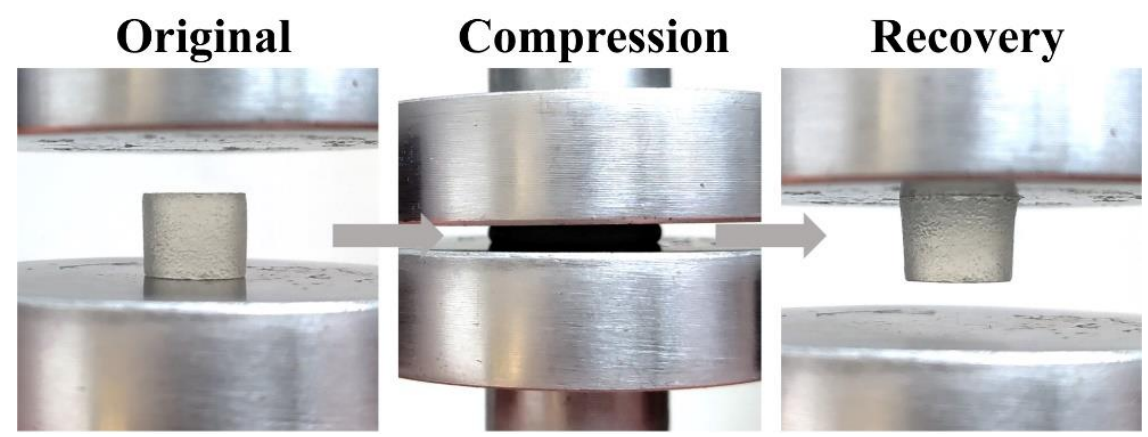

Figure S5. Photographs of dynamic compression process of AP2 hydrogel. 

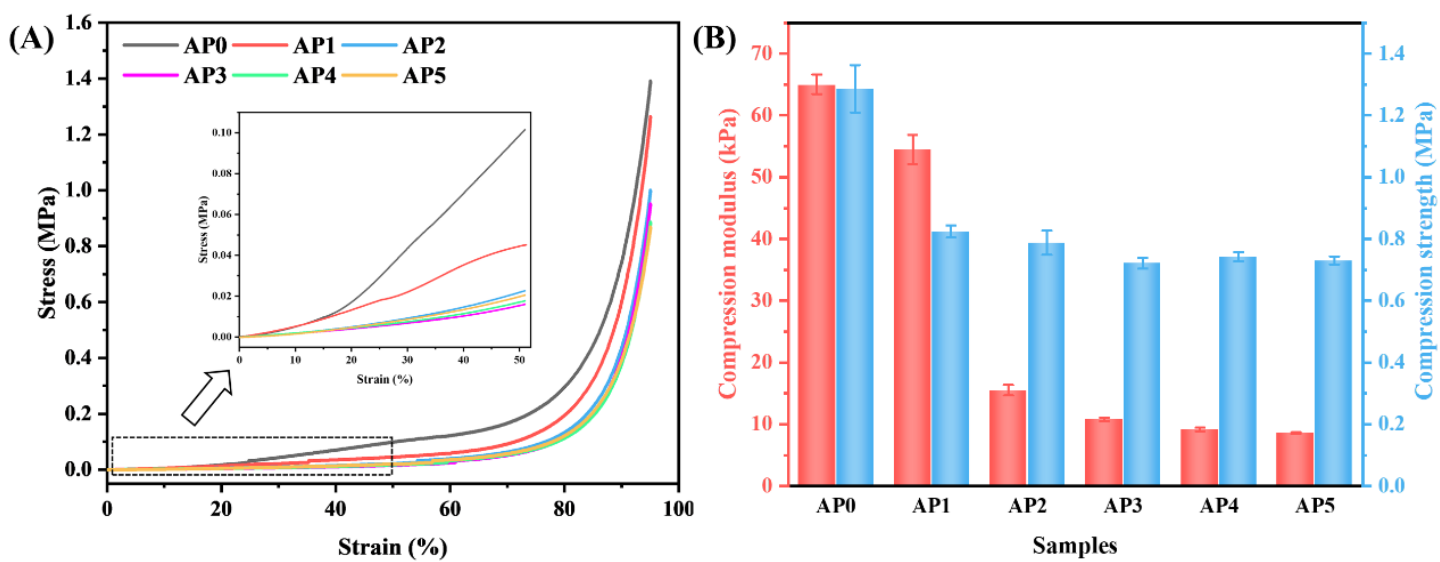

Figure S6. (A) Compression stress-strain curve and (B) compression modulus and strength at $90 \%$ strain of diverse hydrogels.

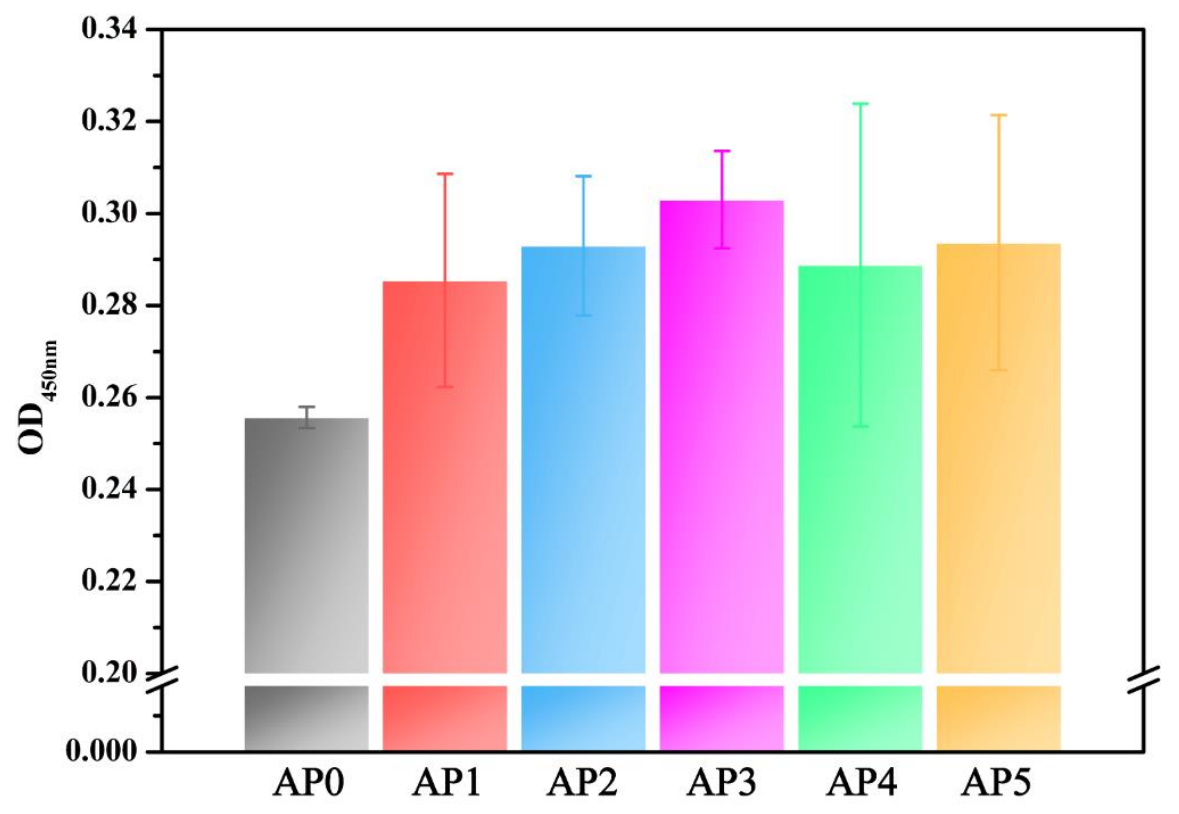

Figure S7. $\mathrm{OD}_{450}$ (absorbance at $450 \mathrm{~nm}$ ) of MC3T3-E1 cells on diverse hydrogels determined by CCK-8 assay after being cultured for $5 \mathrm{~h}$. 


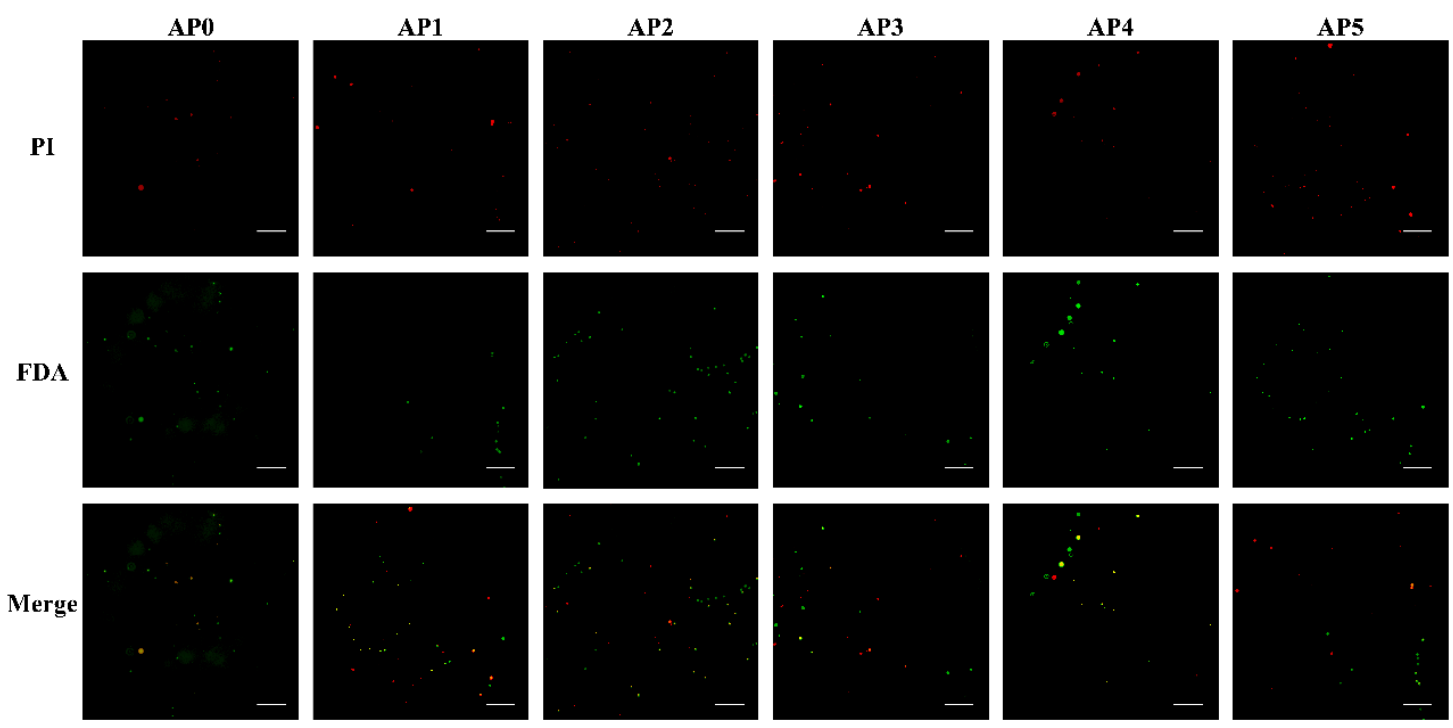

Figure S8. MC3T3-E1 cells on hydrogels after being cultured for $5 \mathrm{~h}$. Live and dead cells were stained by FDA (green) and PI (red), respectively. Scale bar $=50 \mu \mathrm{m}$. 